\title{
Experiences and Perspectives of the Burn Survivors: Inputs for Protocol Development
}

\author{
Maria Jelyn S Garcia BSN, MAN ${ }^{1 *}$ and Jibin Kunjavara MSN, MBA ${ }^{2}$ \\ ${ }^{1}$ Burn Unit, Al-Wakra Hospital, Hamad Medical Corporation (HMC), Qatar \\ ${ }^{2}$ Department of Nursing Research, Hamad Medical Corporation (HMC), Qatar
}

\begin{abstract}
Burn is one of the major culprits in causing morbidity, prolonged hospitalization, disfigurement, and disability to the burn survivors. Several studies have suggested various interventions and management for these burn care challenges. These interventions need to take a holistic perspective of the burn incident and its multiple ramifications, involving the pre- and post-mental health effects, family and significant others' support and most notably, the patient's experiences of pain in relation to the burn experience in general. The objective of this study is to explore the experiences and perspectives of the burn survivors as basis for protocol development. Phenomenological, qualitative approach was employed in this study. Researcher-structured interview guide questionnaires were used for the data collection and ten (10) burn survivors were selected purposively to participate based on the inclusion criteria. Four (4) major themes emerged from the interview narratives: Memories of the Traumatic Event, Burn Challenges and Concerns, Pragmatic Coping Strategies and Goal Directedness. As a result, a burn awareness program can be advocated annually to inform the people of the safety precautions and measures to avoid getting burned. Further education and reiteration on burn pain assessment and management need to be initiated for the nurses to establish standard pain management; regular pain competency can be also integrated in the nurses' continuous educational plan. Patient's pre-discharge training and consistent mental health assessment and reviews can also valuably make the burn care more significant.
\end{abstract}

\section{Keywords}

Burn injury, Burn survivor, Burn survivors' experiences, Burn survivor perspectives, Protocol development

\section{List of Abbreviations}

TBSA: Total Burn Surface Area; PTSD: Post-Traumatic Stress Disorder; PTG: Post-Traumatic Growth

\section{Introduction}

Burn, ranked as a top 15 leading causes of burden globally [1] is defined as a thermal destruction inflicted by plethora of factors as any forms of physical, electrical, and biochemical agents that cause local and systemic implications. The injury is a representation of both physical and psychological assault to the patients that leaves tangible and intangible scars that may be long-lasting and frequently may lead to chronic disability [2]. It is highly regarded as one of the major culprits in causing morbidity, prolonged hospitalization, disfigurement, and disability to the survivors [3].

According to the burn epidemiology undertaken by the World Health Organization (WHO) [4] in 2018, there were 265,000 reported mortality cases annually with the highest prevalence rates in the Western Pacific Region, Eastern Mediterranean and the Southeast Asia Region [1]. However, on the other hand, there has been a remarkable improvement in the patients' chances of survival over the past 20 years in the general populace. Survival rate reaches
95\% of burn-treated patients [5]. Journal of the American College of Surgeons (2018) [6] reported that the mortality rate was ostensibly high in the early years; however, survival chances of the patients improved dramatically due to burn care strides and advancements over the last 30 years.

In comparison, nowadays patients up to 40 years of age who acquired $95 \%$ TBSA tend to survive in contrast to the past years that $50 \%$ body burn killed that same person.

*Corresponding author: Maria Jelyn S Garcia BSN, MAN, Burn Unit, Al-Wakra Hospital, Hamad Medical Corporation (HMC), Doha, Qatar

Accepted: August 07, 2021

Published online: August 09, 2021

Citation: Garcia MJS, Kunjavara J, et al. (2021) Experiences and Perspectives of the Burn Survivors: Inputs for Protocol Development. J Nurs Pract 4(2):327-333 
Citation: Garcia MJS, Kunjavara J, et al. (2021) Experiences and Perspectives of the Burn Survivors: Inputs for Protocol Development. J Nurs Pract 4(2):327-333

Meanwhile, a retrospective analysis conducted in Qatar by Al-Tamimi and Al- Ghoul [7] in 2008 that revealed males (68\%: 1816/2,667) as to be more affected by burn, of which are mostly are expatriate labourers. Minority of $27.4 \%$ formed by the Qatari nationals. In general, causes of burn are flame and scalds comprising of $51.3 \%$, and $41.3 \%$ respectively. Most burn patients $(83.7 \%$ ) had $15 \%$ TBSA burns with a $10.52 \%$ mean. Survival rate is impressively high since mortality rate was only $0.7 \%$ that mostly caused by flame burns (88.9\%).

Extreme burns induce physiologic reactions that influence almost every organ system. Notable characteristics as insulin resistance, muscle wasting and metabolic changes as hypermetabolism are reckoned to continue for many years post-burn [8]. Furthermore, some studies also shown that burn survivors are at risk of diseases as cancer, cerebrovascular disease and infection [9]. In addition, Khadilkar, et al. [10], Ashfaq, et al. [11], Amiri H [12], Mason, et al. [13] cited that the psychological adversities as pain, anxiety, depression, post-traumatic stress disorder, disturbance in bodily ideals disfigurement, alienation, and financial pressure due to prolonged hospitalization are the most common psychological problems encountered by the patients. Burn survivors highly attribute changes in their physical appearance $[14,15]$ and lasting pruritus $[16,17]$ as another major stressors post-injury. These changes may include skin tightening and scarring, skin pigmentation, or even amputations in cases of limb necrotic burn that affect their self-esteem and body image [18].

Survivorship is a major challenge to every burn survivor. The survivors encounter various consequences in their return to normal life as they respond to all the difficult dimensions embedded in the nature of the injury and its rehabilitation. Their journey does not stop in the mere returning to their society; however, only the start of it [19]. In lieu of this, defining and acknowledging the problems other than the physical disabilities can further enhance beneficial outcomes for the burn survivors in achieving a bearable post-burn life.

In spite the advancement modern world offers now, available protocols to assist in the complete rehabilitation and transition of the survivors remain significant challenges as there are still perceived deficits in the consistency and primacy for a holistic burn care approach. Protocol enhancement is indeed crucial in creating effective platforms in the procurement of a rigorous and comprehensive burn care approach.

Several studies have suggested various interventions and management for these burn care challenges. According to Cleary, et al. [20] and Heydarikhayat, et al. [21] pre- and postassessment review of mental health for burn patients need to be facilitated for early recognition of the right support at the right time. According to the Standards and Strategy for Burn Care recommendation, burn patients must preferably be screened for any early psychological adversities within the first four days of the admission. The therapeutic role of nursing staff in burn care is also regarded as a relevant key to therapeutic progression of the survivors $[22,23]$. In order to enhance nurses' interaction with patients, it is recommended that the managers must initiate enhancement programs and trainings for these nurses in lieu with empowering skills and knowledge in the fields of customer care and relations, professionalism and emotional intelligence [24,25].

In this context, it is very important to study the experiences and perceptive of burn survivors to retrieve the real information for giving insights to the health care managers to revise and develop more holistic and rehabilitative system of management protocol among the burns patients.

\section{Materials and Methods}

\section{Research design and sample}

The phenomenological qualitative research design was adopted for this study. A qualitative approach helps to explore the burn survivors' experiences and perspectives to give appropriate inputs for protocol development in managing burn patients. The aim of the researchers is to describe the situation or phenomenon as accurate and authentic as possible, disregarding any pre-biases and preconceived assumptions on the respondent's experiences and reactions to the situation [26]. This research design immensely provided in-depth information on the burn survivor's experiences and their own perspectives in multi-dimensions involved in their burn injury and rehabilitation.

Ten (10) participants ( 2 females and 8 males) were purposively selected to collect the data. All the participants are from Asian countries. Researchers ensured the representation from different nationalities before data collection. Participants selected based on the criteria: 1) patient admitted with total burn surface area (TBSA) of $15 \%$ and above and later been discharged from hospital; 2) ages 18 and above, where as participants who were not willing to give consent were excluded from the study.

\section{Study area and period}

The Preliminary data was collected from the hospital in AlWakrah, one of the tertiary hospitals under Hamad Medical corporation. The face-to-face interviews were conducted in the industrial areas in Qatar and some were outside the participants' workplace after obtaining the informed consent. The interviews were conducted from January to February 2021.

\section{Data Gathering Procedure}

After getting permission from the concerned authorities in Hamad Medical Corporation. The participants were chosen by the inclusion criteria. The selected participants belong to different nationalities like Filipino (2), Indian (2), Nepalese (2), Bangladeshi (3), Sri Lankan (1) based on the admission in the Hospital. Initial or verbal consent were sought through phone then interview date was arranged.

Eight participants are industrial laborers and the remaining two are cashier and waitress respectively. Researchers approached company authorities to get permission to conduct face to face conversation with the participants. The 2 female participants were interviewed outside their workplace (out of duty hours) and no managers were involved. The line mangers of eight participants agreed to conduct interview in congruent to the strict Covid-19 Protocol. Great observance 
of the coronavirus precautionary measures and full disclosure were made prior obtaining the written informed consent from the participants. During the consent, the researcher clarified the objectives of the study. Further, no monetary benefits were offered but tokens of appreciation were given to the participants upon the completion of the study.

Face-to-face interviews with the participants were facilitated with the researcher semi-structured interview guide questions. Privacy and confidentiality were ensured to the participants. During the interviews, the researcher recorded all interview transcripts which lasted for 30-45 minutes. Interview narratives were immediately transcribed and examined within 24-72 hours of post-interview to ensure accuracy of the transcribed data. Permission for future appointments and follow-up interviews were arranged in accordance to participant's availability as stipulated in the consent.

\section{Data Analysis}

Giorgi's method was used in this study as a guide in the analysis of data gathered from the interview. It follows four sequential steps as presented below:

\section{Step 1. One reads through the whole transcript to get the sense of the whole}

In this step, an interview was administered to each participant of the study to get the entire picture of the scenario. To become more acquainted with the words and the order in which they were said, each transcript was read several times. To ensure that the researchers are involved with the words of the participants, caution was taken throughout without any effort to translate the meaning.

\section{Step 2. To evaluate the natural 'units of sense' as articulated by the participant}

In this step, the researchers read and re-read the transcripts of the interview and then identified the best part in the interview about the participant's experiences and perceptions about his injury. To find a core theme for every unit, each transcript was read several times. It is important to reiterate that, as it appears to the naked eye, the theme merely illustrated the main issue of each unit, it did not seek to apply it to the study or interpret its significance.

\section{Step 3. Cross-examination with respect to the basic intent of the report}

This phase focused into both the natural units and the central themes and asked the question "what does this tell me about experiences and perspectives of the burn survivors".

Step 4. The themes have been outlined; an effort was made to bring themes together in an essential descriptive statement and nonredundant themes

This step included recognizing of experiences common to all participants in the study. All emerging themes were identified.

\section{Results}

\section{Major theme 1: Memories of the traumatic event}

This theme describes how the survivors recalled their experiences in acquiring the burn injury. This specifically entails the precise specifics as to how the survivors got injured and their initial reaction on the burn scene. The initial feelings on how the burn injury affected them also vary as the reaction to stimuli as injury is extremely individualized.

Sub-theme 1: Acquired by an accident: This sub-theme describes the acquisition of the burn injury through an accident as narrated by the survivors. The participants experienced burn injury because of an accident. All happened both at home and in their workplace. Most have mentioned that the incident occurred unexpectedly thus they were not able to avoid the accident. Some may have noticed potential signals of the danger (as gas leak, sparks, and exposed wiring), however, did not seem to mind them and still pursued their risky tasks. These burn accidents may have occurred only in split seconds, however still left them severely injured. Fortunately, amidst the terrifying incident, they all managed to flee from the scene and able sought for emergency assistance.

Sub-theme 2: Feeling of imminent death: This subtheme defines the initial thought of most of the survivors when they had the injury, especially those who were gravely affected. It is the abrupt conception upon acquiring the injury as they strived to flee from the harrowing scene. Acquiring traumatic and serious injuries as burn made majority of the participants to reckon about the great possibility of dying on the burn scene itself. The feeling of impending doom was evident upon getting inflicted by the injury. They initially felt the intuition and perplexity right on the incident that drawn them into a conclusion that they may not able to survive that life-threatening situation. Burn incidents unfolded in just split seconds that left the participants in doubt of their own ability to survive or almost given up their hope of surviving. They had even lost consciousness that could barely remember the next things that had happened to them.

\section{Major theme 2: Burn challenges and concerns}

This theme summarizes the challenges and main concerns of the burn survivors when they had the burn injury from the initial course of their treatment until they have finally been discharged home.

Sub-theme 1: Physical impacts: This sub-theme focuses on the physical adversities encountered and still being experienced by the participants from the time of their treatment up to the present.

Sub-category 1: Excruciating pain: This sub-category reflects on how the burn survivors describe the pain they have encountered during their treatment. Pain is perceived by the participants as one of their major concerns during the treatment. It has been described as horrible, unbearable, unexplainable, daunting and something they could never imagine encountering in their lives. It was described as the most painful amongst any other injury. It massively gave 
Citation: Garcia MJS, Kunjavara J, et al. (2021) Experiences and Perspectives of the Burn Survivors: Inputs for Protocol Development. J Nurs Pract 4(2):327-333

enormous pessimistic feelings that made them reluctant to engage well in the dressings and any other procedures, and often brought harrowing nightmares.

Sub-category 2: Post-burn scars and contractures: This sub-category refers to the physical disabilities that the participants have acquired post-burn injury. These deformities include post-burn scarring and contractures. These restrictions started during the initial phase of their rehabilitation, which continued until post-discharge. The severity of the injury is a great factor on such, most of which had trouble in performing tasks especially when they went back to their usual function pre-burn. Multi-modal interventions as topical treatments, scar revision and contracture release may have already been facilitated, nevertheless still left them with sort of discomfort and lasting disabilities. These burn aftermaths gave the participants subsequent paucity in the movement and the dearth feeling of going out and get exposed publicly.

Sub-theme 2: Psychological impact: This sub-theme describes the mental and emotional adversities this burn injury brought to the survivors. They have narrated own struggles as how they suffer psychologically during their course of treatment until the very moment that they are physically healed. Majority of the participants have encountered inevitable psychological struggles that were brought by the burn incident. These adversities as commonly stated include anxiety due to procedural (surgery and dressing) and burn pain, depression and post-traumatic stress disorder (PTSD) that brought them into sorts of hallucinations and sudden nightmares. Having these, immensely impacted their treatment and recovery. On the contrary, getting depressed and traumatized upon seeing their scars and recalling vividly the memories of their incidents had a huge wallop in adjusting to their present life and functions post-burn.

Sub-theme 3: Financial distress: This sub-theme relates to the financial challenges the participants have undergone and continuously been encountering as they were on their journey in treatment and recovery. These financial ramifications greatly contributed to their stress, especially when they faced longer hospitalization and treatment; and felt that things are not really quiet the same as before financial-wise.

Some of the participants reckoned that their injuries have a profound impact on their financial status, particularly for those who have sustained grave burn injuries which restrict them in performing their usual tasks and functions as efficiently as before. Though, burn treatment and medications were free of charges in the hospital, regardless of their country's visa status; still others still worried much about the dramatic decrease in the amount they normally earn for a living when they resume back to their jobs due to their medical and physical burn complications. They were feeling fortunate as their company still provided them their salary during their hospitalization; however, some extra jobs that they would usually do before could no longer be done now a days. In addition, due to the physical disabilities, there are also medical needs that can no longer be availed for free since they are already discharged.

Sub-theme 4: Concerns about the services and the staff: This sub-theme explains the participants' perception in some of the aspects of the burn care during their treatment course and their concerns to some of the staff that gave an impact to their pain management, discharge procedure, and any other burn modalities involved. These concerns depict issues primarily related to pain management, discharge planning and procedures, communication issues with the staff, even the need for a consistent psychological assessment and interventions that essentially may spearhead change and quality improvement initiatives if addressed properly. It has been said that patients are mostly likely become more engage and interested in their own treatment and recovery if they are satisfied with the care they received from the team.

\section{Major theme 3: Pragmatic coping strategies}

This theme elicits the different coping strategies acquired and developed by the participants as they encountered various burn challenges in the new transition of their lives.

Sub-theme 1: Seeking strength in prayers: This sub-theme deals with how prayers and faith helped the participants in their coping. No matter how distinctive their faith may be, they have turned into God as a great source of their strength. The participants had high regard with God and used the essence of prayers when dealing with their negative thoughts. They claimed that they were granted such kind of suffering due to a special reason. They highlighted that turning all their worries and pain to God profoundly changed their lives and their perspectives. Some may have initially put blame towards Him for their suffering, however still realized that they were still been blessed for another chance to live.

Sub-theme 2: Support system: This sub-theme depicts how valuable the support system of the participants in facing their struggles during treatment and recovery. The participants commonly verbalized that good support system immensely assisted them in their coping from their disease process. Though their families were not physically present for them, distance did not preclude them from rendering support to the participants. It was a tremendous relief that the use of technology paved the way in connecting with their families anytime they needed them. Apart from that, presence of their friends was indeed a supporting factor too. The latter supported them in the absence of their families. They became their confidante, spiritual mentors, and literally the "wind beneath their wings". Healthcare workers on the other hand, especially the nurses play integral role in the burn treatment and rehabilitation of these patients. Therefore, it is extremely valuable that when planning of the burn care, understanding of the unique needs of the patients must be taken into great account.

Sub-theme 3: Diversional activities: This sub-theme refers to the diversional activities being utilized by the participants to cope up with their pain and struggles during their treatment and recovery. The participants narrated different activities that they have utilized during their burn treatment until during their rehabilitation process. Technology plays a vital and relevant role in allowing them to redirect their focus. With the use of devices, they were able to divert their pain by watching the television and mobile. Inspirational videos and learning new things as English language and burn wound 
Citation: Garcia MJS, Kunjavara J, et al. (2021) Experiences and Perspectives of the Burn Survivors: Inputs for Protocol Development. J Nurs Pract 4(2):327-333

dressing at home were just of the things being browsed by the participants to empower their knowledge and fight bore some while staying longer in the hospital bed. Moreover, reading some news and books also kept them updated during their hospitalization. Achieving independence (as early walking ad roaming around the room and unit) as early as possible also redirected their negative thoughts and feelings.

Sub-theme 4: Self-efficacy: This sub-theme explains the integral role of self-efficacy in the treatment and recovery of the participants. The participants have expressed strong will in conquering their battle against the adversities and mishaps brought by the burn incident. They may initially feel the hesitation and self-doubt; some even expressed self-blame; however gradually realized the essence of selfmotivation and self-resilience in their recovery. They found strength not only from the others' support and other coping strategies, but through self-motivation as well. Self-efficacy enabled them to employ effective coping skills during the tough times. This became an essential tool or variable in the gradual successful adaptation to all forms of changes postburn. It is also worth mentioning that having the sense for self-efficacy valuably helped the participants to deal with the long-term disabilities and problems, most importantly in their psychological rehabilitation.

\section{Major theme 4: Goal-directedness}

This theme postulates the participants' ability to foresee and prepare for their future goals and dreams years after the incident. One of the great commonalities amongst the participants is the ability to formulate goals for themselves years after the burn incident, considering the challenges they have endured. Planning for long-term goals as reuniting back home to their families, making business and successfully achieving physical functions, aesthetic considerations, and even becoming a model and living inspiration for the other burn victims are part of these goals as they continually adjust to their post-burn lives. Setting goals by and for themselves enables them to restore self-worth and self-esteem. Support system as the burn care team are essential for the initial burn management and treatment during hospitalization, nevertheless the ability to formulate goals by themselves is vital for their own post-traumatic growth.

\section{Discussion}

In our study, survivors have narrated enormous concerns in lieu of their experiences and perspectives on their treatment and recovery. Four themes emerged from the pronouncements of the burn survivors about their burn experiences and perspectives namely: Memories of the Traumatic Event, Burn Challenges and Concerns, Pragmatic Coping Strategies and Goal Directedness. Theme 1refers to how the burn survivors have sustained the injury. It has been described as accidentally acquired and most have which initially felt imminent death. According to the World Health Organization (2018) [4], burn accidents are significant concerns that need emergency attention worldwide. These accidents occur in different settings, but they have conducted a 10-year survey that summarized the usual location of a burn accident; most of which are happening at home and at workplace. Furthermore, Martins, et al. [27] mentioned the enormous factors contributing to such burn accidents. They have discussed stressors as interpersonal and extra personal factors involved. The intensity of the activity pace, disproportionate workload and stress emerged as interpersonal stressors. And extra personal stressors such as old equipment and faulty handling of instrument and precarious job status are highlighted. It has been reiterated that regardless of the usual location of the burn accidents, burn per second is very preventable. American Burn Association is usually launching yearly National Burn Awareness week that converses major domains as burn awareness, hazard protection and prevention to the public to lessen the risk of any burns.

In theme 2, the survivors have recounted plethora of challenges and concerns they have encountered and raised from their hospitalization up to post-discharge state. They have enumerated burn impacts on their physical, psychological, and financial status that immensely affect their normal functions, thoughts and perceptions. Moreover, they have also gathered concerns about the services they have received and the staff they have encountered. According to McLean, et al. [15] changes in the body due to burn damage tended to be just one of the main difficulties that these survivors encountered in integrating combined traumatic events. Physical impacts as excruciating pain and presence of lasting scars and contractures triggered these survivors to experience psychological adversities as PTSD, anxiety and depression. Financial distress is also something the relevantly considered as culprit for such mental discomfort. More so, Lotfi, et al. [28] revealed that most of the victims discussed significant issues on their discharge experience that primarily centered on their convenience in performing usual tasks upon returning home and their post-discharge follow-ups and care coordination. Staff communication has also been highlighted as predictor of the patient's perception of quality care.

Pragmatic coping strategies helped the burn survivors in their coping and adapting to their unprecedented condition and functions. Included in these strategies are seeking strength in prayers, presence of the support system, utilization of diversional activities and the vital role of the survivors' self-efficacy. Employing such strategies bolstered them in gradually combating the hurdles spearheaded them. Jibeen, et al. [29] mentioned that burn victims who rely on moral or religious values in their coping may display greater post-traumatic growth (PTG). Studies indicated that the role of the family and friends is very important in recovering the physical and mental control of the survivors and at the same time increasing their power. Healthcare workers on the other hand, especially the nurses play integral role in the burn treatment and rehabilitation of these patients [30]. Most of the survivors find convenient activities to cease them from worrying about the unfortunate incident that has changed their lives. These activities entail reading books, doing chores, work addiction, and any other effective methods. Positive coping habits offer extra tools that help them in coping with the stressor's demand. Furthermore, efficient coping 
Citation: Garcia MJS, Kunjavara J, et al. (2021) Experiences and Perspectives of the Burn Survivors: Inputs for Protocol Development. J Nurs Pract 4(2):327-333

behaviour's offer tenacity in the reduction of detrimental burn consequences; subsequently improving growth [31]. Studies found that self-efficacy also has a significant impact in counterfeiting negative effects of a traumatic event as burn [32].

Lastly, amidst the setbacks these survivors have faced due to the burn injuries, they nevertheless continued to direct their lives and set goals for their future interventions. Part of these goals are reverting to their families in their home origin, anticipating aesthetic consideration to manage their scars and physical disfigurements and successfully performing physical tasks normally, and becoming living inspiration for the other burn victims. According to Wallersteiner [33], it is extremely useful for the burn victims to set goals for themselves as an essential method for coping from their injury, pain and burn challenges. It provides them a sense of accountability in planning for their own care and rehabilitation and ponder on the obstacles that may hinder them reaching their goals.

\section{Limitation}

Even though study retrieved all possible phenomenological themes, researchers enlist limitations that majority of the study participants were industrial labourers. The demographic profiles and standard of living may be quite different in other communities. It may influence the participants psychological state of response towards the questions. In addition to that, majority of the participants were males than females that also can be considered as limitation of this study.

\section{Conclusion and Recommendation}

The study findings give wider venue for improvement in terms of burn nursing and management in general. The narrated experiences and perspectives of the burn survivors open great opportunities in the enhancement and development of essential protocol that will provide sophisticated and comprehensive burn care approach. Nursing administration can gain knowledge in the right prescription of care and management for the burn patients. Based on the findings, recommendations were formulated. A burn awareness program can be advocated annually, this will include fire and safety prevention at home and in the workplace to inform the people of the safety precautions and measures to avoid getting burned. Further education and reiteration on burn pain assessment and management needs to be planned and initiated for the nurses to establish standard pain management; regular pain competency can be also integrated in the nurses' continuous educational plan. Furthermore, to address the concerns of the burn survivors about increasing independence and confidence prior discharge, a pre- discharge training can be initiated days or weeks before the expected discharge which may include independent self-care and activities of daily living, application of own topical medications, ambulating, and doing own exercises. Mental health assessment and review should also consistently be integrated in the burn care. Psychological visits should be regarded from the early hospitalization until clinic follow-ups to review the psychological status of the survivors especially when they return to the society. In addition, regular case conference be integrated amongst the burn multi-disciplinary team to imperatively review the progress of the patients whilst admitted. The researcher and future researchers may conduct similar studies by using the results of the present study.

\section{Declaration of Conflicting Interests}

The authors declare that there is no conflict of interest.

\section{Funding Statement}

This research received no specific grant from any funding agency in the public, commercial, or not-for-profit sectors.

\section{Ethical Consideration}

The study obtained permission from Institutional Review Board of Hamad Medical Corporation with the number (MRC01-20-1194). This study conducted based on the principles of Belmont Report and obtained written consent from all participants before the data collection.

\section{References}

1. Peck M (2019) Epidemiology of burn injuries globally. Up to Date.

2. Jain A, Rathore S, Jain R, et al. (2017) Assessment of the depression and the quality of life in burn patients seeking reconstruction surgery. Indian J Burns 23: 37-42.

3. Ali S, Hamiz-ul-Fawwad S, Al-ibran E, et al. (2016) Clinical and demographic features of burn injuries in Karachi: A six-year experience at the burns centre, civil hospital, Karachi. Ann Burns Fire Disasters 29: 4-9.

4. (2018) World Health Organization. Burn factsheets.

5. Gauglitz G, Williams F (2020) Overview of the management of the severely burned patient. Up To Date.

6. American College of Surgeons (2018) Burn specialists report a dramatic increase in burn injury survival over the past 30 years.

7. Al-Tamimi N, Al-Ghoul A (2008) Burn epidemiology in Qatar: A retrospective analysis of 2,667 patients. JEMTAC 8: 100-108.

8. Nielson C, Duethman N, Howard J, et al. (2017) Burns: Pathophysiology of systemic complications and current management. J Burn Care Res 38: e469-e481.

9. Duke J, Vetricheyvel T, Randall S, et al. (2018) A populationbased comparison study of the mental health of patients with intentional and unintentional burns. Burns Trauma 6: 31.

10. Khadilkar N, De Sousa A, Jain M (2017) Burn-related factors affecting anxiety, depression and self-esteem in burn patients: An exploratory study. Ann Burns Fire Disasters 30: 30-34.

11. Ashfaq A, Lashari U, Saleem S, et al. (2018) Exploring symptoms of post-traumatic stress disorders and perceived social support among patients with burn injury. Cureus 10: e2669.

12. Amiri $H$ (2015) The emotional impact of burns on the patient. Burn Injury Resource Center.

13. Mason S, Nathens A, Byrne J, et al. (2017) Association between burn injury and mental illness among burn survivors: A population-based, self-matched, longitudinal cohort study. J Am Coll Surg 225: 516-524.

14. Goei $H$, Van der Vlies $H$, Tuinebreijer WE, et al. (2017) Predictive validity of short-term scar quality on final burn scar outcome using the patient and observer scar assessment scale in patients with minor to moderate burn severity. Burns 43: 715-723. 
15. McLean L, Rogers V, Kornhaber R, et al. (2015) The patient-body relationship and the "lived experience" of a facial burn injury: A phenomenological inquiry of early psychosocial adjustment. J Multidiscip Healthc 8: 377-387.

16. Finnerty C, Jeschke M, Branski L, et al. (2016) Hypertrophic scarring: The greatest unmet challenge after burn injury. Lancet 388: 1427-1436.

17. Chung B, Kim H, Jung M, et al. (2020) Post-burn Pruritus. Int J Mol Sci 21: 3880.

18. Moi A, Haugsmyr E, Heisterkamp H (2016) Long-term study of health and quality of life after burn injury. Ann Burns Fire Disasters 29: 295-299.

19. Zamanzadeh V, Valizadeh L, Lotfi M, et al. (2015) Preserving selfconcept in the burn survivors: A qualitative study. Indian J Palliat Care 21: 182-191.

20. Cleary M, Visentin D, West $S$, et al. (2017) The importance of mental health considerations for critical care burns patients. J Adv Nurs 74: 1233-1235.

21. Heydarikhayat N, Ashktorab T, Rohani C, et al. (2018) Effects of post-hospital discharge follow-up on health status in patients with burn injuries: A randomized clinical trial. Int J Community Based Nurs Midwifery 6: 293-304.

22. Mula J, Estrada J (2020) Impact of nurse-patient relationship on quality of care and patient autonomy in decision-making. Int J Environ Res Public Health 17: 835.

23. Hartley S, Raphael J, Lovell K, et al. (2020) Effective nursepatient relationships in mental health care: A systematic review of interventions to improve the therapeutic alliance. Int J Nurs Stud 102: 103490.
24. Yarney L, Yeboah E, Hansen-Garshong R (2019) Experiences of burn patients in Ghana: A qualitative study at the Korle-Bu teaching hospital in Accra. Indian J Burns 27: 49-56.

25. Kaihlanen A, Hietapakka L, Heponiemi T (2019) Increasing cultural awareness: Qualitative study of nurses' perceptions about cultural competence training. BMC Nursing 18: 38.

26. Christensen M, Welch A, Barr J (2017) Husserlian descriptive phenomenology: A review of intentionality, reduction and natural attitude. J Nurs Educ Pract 7: 113.

27. Martins M, Echavarria-Guanilo M, Silveria D, et al. (2015) Risk perception of work-related burn injuries from the workers perspective. Texto \& contexto enferm o 24: 1148-1156.

28. Lotfi M, Ghahremaneian A, Aghazadeh A, et al. (2018) The effect of pre-discharge training on the quality of life of burn patients. J Caring Sci 7: 107-122.

29. Jibeen T, Mahfooz M, Fatima S (2018) Spiritual transcendence and psychological adjustment: The moderating role of personality in burn patients. J Relig Health 57: 1618-1633.

30. Fei H, Zhou Q, Zhao Z (2016) Effect of perceived social support and dispositional optimism on the depression of burn patients. J Health Psychol 21: 1119-1125.

31. Kool M, Geenen R, Egberts M, et al. (2017) Patients' perspectives on quality of life after burn. Burns 43: 747-756.

32. Bosmans $M$, Hofland $H$, De Jong A, et al. (2015) Coping with burns: The role of coping self-efficacy in the recovery from traumatic stress following burn injuries. J Behav Med 38: 642-651.

33. Wallersteiner R (2020) James Partridge: Founder of the UK charity changing faces. BMJ 370.

DOI: $10.36959 / 545 / 401$

Copyright: (c) 2021 Garcia MJS. This is an open-access article distributed under the terms of the Creative Commons Attribution License, which permits unrestricted use, distribution, and reproduction in any medium, provided the original author and source are credited. 